\title{
The generalization of an R-S* expectancy in discrimination learning
}

\author{
TERRY A. ROOT and HENRY A. CROSS \\ Colorado State University, Fort Collins, Colorado
}

\begin{abstract}
Two groups of rats were trained in a horizontal-vertical discrimination in a modified Grice box after either $100 \%$ or $50 \%$ reinforcement for 200 jumps to a single gray stimulus. A control group received only the discrimination. Animals receiving $100 \%$ reinforcement in the jumping situation discriminated more quickly than did the controls or those receiving 50\%. Results supported those of Purdy and Cross (1979) and strengthened the case for R-S* expectancy learning (Bolles, $1972,1975)$. Additionally, these data indicate that $R-S *$ learning can generalize.
\end{abstract}

Reid (1953) demonstrated that rats overtrained on a discrimination problem learn the reversal of the task in fewer trials than do subjects trained only to criterion on the initial discrimination. Attempts to explain this phenomenon, the overlearning reversal effect (ORE), have given rise to two main lines of thought: (1) that of attention theorists, who argue for specific transfer from original discrimination to reversal (e.g., Sutherland \& Macintosh, 1971), and (2) that of those who pose a more general transfer effect (e.g., Hall, 1973; Reid, 1953).

Sutherland and Macintosh (1971), with their notion that overtraining in a discrimination task strengthens an "analyzer" relevant to that task, would generally argue that transfer to a subsequent task, for example, discrimination reversal, would facilitate the later task only if the analyzer remained the same, that is, relevant to both the original and transfer tasks. Reid's (1953) theory, on the other hand, can accommodate empirical findings (Hall, 1974 ) in which positive transfer is identified even though the "dimension" of the discrimination is switched as organisms are moved from the original to transfer situations. Reid's theory can be made to account for such general transfer findings by the use of his notion of a gradual development of a "response of discriminating" that parallels the specific choice responses that the organism learns to the positive and negative discriminanda. Basically, Reid maintained that late in learning, and particularly during overtraining, the animal becomes increasingly proficient in attending to and responding to differences between or among the discrimination stimuli and, since this is a pattem of behavior that should facilitate any subsequent discrimination learning, it is not critical that the relevant dimension remain fixed as one moves from discrimination to some reversal or subsequent discrimination-learning task.

Although the specific attention and general attention theorists disagree about what an organism learns during

Requests for reprints should be addressed to: Henry A. Cross, Department of Psychology, Colorado State University, Fort Collins, Colorado 80523. overtraining and can subsequently transfer, there is one point of similarity. Both groups agree that the development of specific analyzer strength and/or the strengthening of a "response of discriminating" take place only in the context of a discrimination proper, and, for this reason, neither group can readily deal with findings that pretraining on a single stimulus from another dimension facilitates subsequent discrimination learning (Purdy \& Cross, 1979, Experiment 1) or that such single-cue training interpolated between discrimination and discrimination reversal enhances reversal (Purdy \& Cross, 1979, Experiment 2).

Purdy and Cross (1979) argued that, in their singlecue situations, it was possible for an animal to learn an R-S* contingency or expectancy (as discussed by Bolles, 1972, 1975) and that this learned response-reinforcement relationship explains, in large part, the facilitative effect that overtraining may have on a subsequent discrimination task-even one that involves a changed dimension.

A question of interest, and one that is the focus of the current investigation, centers around the degree of the response change that can be tolerated as one moves the organism from single-cue training to discrimination proper. In the Purdy and Cross experiments, the jumping response was common to both the single-cue pretraining and the subsequent discrimination. In the present study, the initial or pretraining experience involved jumping to a single-cue, whereas the later discrimination task involved a different response and apparatus, that is, walking/running in a modified Grice box.

\section{METHOD}

\section{Subjects}

The subjects consisted of 15 male hooded rats obtained from Research Animals, Inc., Braddock, Pennsylvania. All subjects were approximately 85 days of age at the start of training and were individually housed with water available ad lib.

\section{Apparatus}

A modified Lashley jumping stand and a modified Grice box were used. The single-window jumping stand (SWJS) was identical to the one described by Purdy and Cross (1979). The Grice 
box consisted of two main parts: the goal area and the start box. The goal area was essentially a box $25 \mathrm{~cm}$ in length $x 48.5 \mathrm{~cm}$ in width $\times 20 \mathrm{~cm}$ in height, which was divided into three equalsized goal boxes each $25 \times 15 \mathrm{~cm}$. At the back of each goal box was a 3 -cm-diam food cup. A tongue and groove enabled the start box to slide back and forth on the goal box, allowing only two of the three entry doors to be visible at one time.

The start box was $38.5 \mathrm{~cm}$ in length $\mathrm{x} 20 \mathrm{~cm}$ in height. At the end away from the goal area, the width was $22.5 \mathrm{~cm}$ and increased to $32 \mathrm{~cm}$ where the start box and goal area overlapped. A piece of Plexiglas, $5 \times 3 \times 20 \mathrm{~cm}$, was placed upright in order to separate the two visible doorways. The goal box doors, $11.5 \mathrm{~cm}$ square, served as the stimulus objects. The openings for the doors were $10.5 \mathrm{~cm}$ square. Rats placed at the narrow end of the start box ran to either a horizontally or a vertically striated card (black and white striations, each $7 \mathrm{~mm}$ wide).

\section{Procedure}

All rats were placed on a food-deprivation schedule, 10-12 g of Purina laboratory chow per day, 10 days prior to being trained. During this time, they were each handled for $5-6 \mathrm{~min}$ daily. All subjects were then given 2 days ( $15 \mathrm{~min}$ per day) of habituation in the SWJS and were assigned randomly to one of three groups. The rats in the first group, Group SWJS-100, were given 10 jumping trials per day for 20 days to a medium gray card, for a total of 200 trials, and were rewarded four $45-\mathrm{mg}$ Noyes pellets on $100 \%$ of these trials. The second group of rats, Group SWJS-50, were also given 10 trials per day to the same card for 20 days and were rewarded randomly on $50 \%$ of these trials. The rats in the third group served as the controls; they received no pretraining experience in the SWJS beyond the habituation experience.

Following pretraining, all groups, Groups SWJS-100 and SWJS-50 and the control group, were taught the H-V discrimination in the Grice box. They received 10 noncorrection trials per day; the criterion of learning was any 10 correct responses in a row (including across days). Reinforcement was four $45-\mathrm{mg}$ Noyes regular food pellets. Either two or three subjects from each group were trained with $\mathrm{V}+$, and the remaining subjects experienced $\mathrm{H}+$. The stimuli presented in the Grice box were alternated randomly from left to right by sliding the start box to the left or right. Two restrictions were that each cue appear on each side $50 \%$ of the time and that a given cue remain on any one side for no more than two consecutive trials. There was an intertrial interval of approximately $4 \mathrm{~min}$. A ceiling of 200 trials was established at the beginning of the experiment, and any subject not reaching criterion after this period was given a trials-to-criterion score of 200 .

\section{RESULTS}

A one-way ANOVA procedure showed a significant difference in the mean trials to criterion among the three groups $[F(2,12)=6.41, p<.025]$, and a Newman-Keuls comparison of group means revealed that Group SWJS100 learned the H-V discrimination in fewer trials than did either Group SWJS-50 or the control group, the means of which did not differ significantly. Errors-tocriterion scores also resulted in a significant $F$ ratio $[F(2,12)=6.64, p<.025]$, and subsequent comparisons demonstrated that Group SWJS-100 made fewer errors than did the control group, which in turn made significantly fewer errors than did Group SWJS-50. The means for trials to criterion and errors to criterion for the groups are shown in Table 1.
Table 1

Means for Acquisition of H-V Discrimination

\begin{tabular}{lcc}
\hline Groups & $\begin{array}{c}\text { Errors to } \\
\text { Criterion }\end{array}$ & $\begin{array}{c}\text { Trials to } \\
\text { Criterion }\end{array}$ \\
\hline SWJS-100 & 31.0 & 81.8 \\
SWJS-50 & 74.8 & 158.4 \\
Control & 64.4 & 147.7 \\
\hline
\end{tabular}

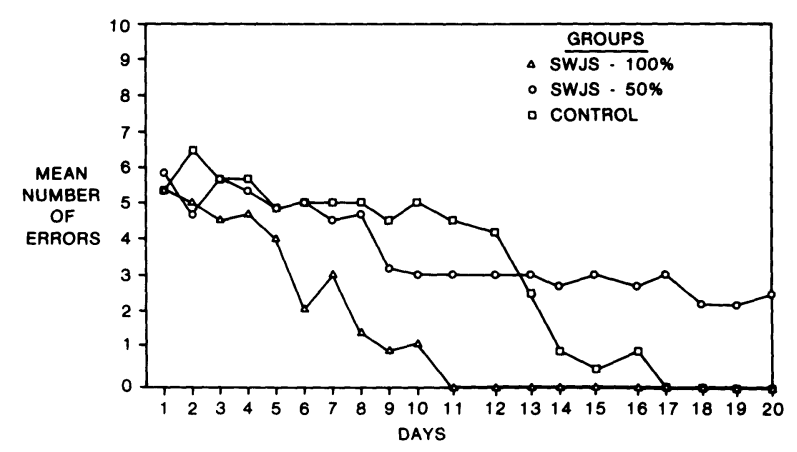

Figure 1. Mean errors by groups over 20 days of discrimination.

A split-plot ANOVA in which the basic datum was the number of errors per daily session revealed that there was a significant group effect $[\mathrm{F}(2,12)=6.64, \mathrm{p}<.025]$, a significant days effect $[F(19,228)=25.62, p<.01]$, and a group $\mathrm{x}$ days interaction $[\mathrm{F}(38,228)=2.431$, $\mathrm{p}<.01]$. These results are depicted graphically in Figure 1 , which clearly shows the nature of this interaction.

The subjects in Group SWJS-100 learned the H-V discrimination with relative ease, and the control subjects, although remaining near chance for a longer time than the Group SWJS-50 subjects, ultimately surpassed the Group SWJS-50 subjects, which, as a group, failed to learn the discrimination within the 200-trial limit.

\section{DISCUSSION}

The results were consistent with those of Purdy and Cross (1979) and provide additional evidence that animal subjects may well learn an R-S* contingency or expectancy in a nondiscrimination context and that such an expectancy can facilitate subsequent discrimination learning. These data are not easily accounted for, either by selective attention theory (e.g., Sutherland $\&$ Macintosh, 1971) or by more general attentional theories (e.g., Reid, 1953), inasmuch as both formulations require a discrimination context for the hypothesized growth of "analyzer strength" or a "response of discriminating." Hall's (1973) response strategy explanation of the ORE also depends upon discrimination experience with both $\mathrm{S}+$ and $\mathrm{S}$ - present.

The present findings support the notion that an acquired R-S* contingency can be remarkably context independent. In the Purdy and Cross (1979) experiments, as well as in the present one, the subjects received their single-cue training on a stimulus that represented a "dimension" other than that employed in their subsequent discrimination or discrimination reversal training. In addition, in the current research situation, both the appropriate instrumental behavior and the type of apparatus 
were changed as the subject was moved from single-cue exposure to discrimination proper. In spite of these changes, the animals receiving $100 \%$ reinforcement with the single cue benefited in the subsequent discrimination learning. The conclusion is that animals are capable of learning R-S* contingencies or an expectancy about how of ten their responses are reinforced and that such an expectancy, once acquired, may generalize. The full extent of such generalization should be the subject of future research.

\section{REFERENCES}

Bolles, R. C. (1972). Reinforcement, expectancy, and learning. Psychological Review, 79, 394-409.

Bolles, R. C. (1975). Learning theory. New York: Holt, Rinehart \& Winston.
Hall, G. (1973). Response strategies after overtraining in the jumping stand. Animal Learning \& Behavior, 1, 157-160.

HaLL, G. (1974). Transfer effects produced by overtraining in the rat. Journal of Comparative and Physiological Psychology, 87, 938-944.

Purdy, E., \& Cross, H. A. (1979). The role of R-S* expectancy in discrimination and discrimination reversal training. Learning and Motivation, 10, 211-227.

REID, L. S. (1953). The development of noncontinuity behavior through continuity learning. Journal of Experimental Psychology, 46, 107-112.

Sutherland, N. S., \& Macintosh, N. J. (1971). Mechanisms of animal discrimination learning. New York: Academic Press.

(Manuscript received for publication December 14, 1983.) 